\title{
Pro-active positioning of a social robot intervening upon behavioral disturbances of persons with dementia in a smart nursing home
}

\author{
Johannes Nauta ${ }^{\mathrm{a}}$, Christof Mahieu $^{\mathrm{a}}$, Christophe Michiels $^{\mathrm{b}}$, Femke Ongenae $^{\mathrm{a}}$, Femke De Backere ${ }^{\mathrm{a}}$, Filip De Turck ${ }^{\mathrm{a}}$, \\ Yara Khaluf ${ }^{a}$, Pieter Simoens ${ }^{\mathrm{a}}$ \\ ${ }^{a}$ IDLab, Department of Information Technology, Ghent University-imec, Technologiepark 126, B-9052 Gent, Belgium \\ ${ }^{b}$ Zorabots, Archimedesstraat 17, B-8400 Oostende, Belgium
}

\begin{abstract}
Behavioral disturbances of persons with dementia residing in a nursing home impose a significant burden on other residents and on the care staff. A social robot can provide an adequate technological support tool for the caregivers by approaching a resident that exhibits a behavioral disturbance. In this paper, we focus on how to position the robot in the nursing home, taking into account the profile and location of the residents. We minimize the time between the detection of a behavioral disturbance and the robot having arrived near the resident and starting an interaction scenario. Our algorithm is evaluated using realistic data that was collected during 3 months in two Belgian nursing homes.
\end{abstract}

Keywords: personalized care, smart environments, Internet-of-Robotic-Things, social robotics

\section{Introduction} hof et al., 2016).
Dementia is characterized by a progressive decrease of 32 cognitive capabilities that affects memory, thinking and 33 social abilities severely enough to interfere with daily func- 34 tioning. As age is one of the strongest known risk factors 35 for dementia, the worldwide cost of dementia care services 36 is projected to grow significantly with the aging popula- 37 tion (World Health Organization, 2018; Harper, 2014).

Ambient assisted living technology can reduce costs by ${ }^{39}$ extending the period in which older adults can stay inde- 40 pendent in their home (Demir et al., 2017). Over time, the ${ }^{41}$ care for a person with dementia often becomes too com- ${ }^{42}$ plex to be organized in the home environment, and at a ${ }^{43}$ certain point the person needs to be placed in a nursing ${ }^{44}$ home with guaranteed supervision and specialized facili- ${ }^{45}$ ties (Mller et al., 2017).

The corollary of these societal and technological evolu- ${ }^{47}$ tions is that the care needs of the average nursing home ${ }^{48}$ resident are becoming more complex and that the demand ${ }^{49}$ by the staff for technological support tools is increasing ${ }^{50}$ accordingly (OSullivan et al., 2018). Behavioral distur- ${ }^{51}$ bances (BD) are one of the most prominent manifestations ${ }^{52}$ of dementia, exhibited by a majority of the persons with ${ }^{53}$ moderate to severe dementia living in nursing homes (De- 54 sai et al., 2012; Husebo et al., 2011). BD can take various ${ }^{55}$ forms, including mood disorders, wandering and verbal or 56 physical aggression. These BDs are an important inhibitor 57 of stress to other residents as well as to caregivers (Hazel- 58

Email address: pieter.simoens@ugent.be (Pieter Simoens)
To manage these BDs, one can use pharmacological or non-pharmacological interventions. Pharamacological interventions are used only in acute situations since these treatments do not address the underlying psychosocial reasons and may have adverse side effects (Sadowsky and Galvin, 2012). Many different non-pharmacological therapies are designed to resolve specific BDs by interacting with the residents and without the harmful effects of medical interventions (de Oliveira et al., 2015). Robot-Assisted Therapies are one particular form of non-pharmacological therapy. Many recent studies investigated the effects of various social robots on persons with dementia, such as the robot seal PARO or the humanoid Nao (Sabanovic et al., 2013; Jøranson et al., 2015; Wada et al., 2005; Valent Soler et al., 2015; Inoue et al., 2014). Even with relatively simple interactions such as storytelling, singing a song or performing a dance, a positive effect on the neuropsychiatric symptoms is reported. However, these robots only interact in a preprogrammed manner and it is often too time consuming for the staff to program new therapy sessions on the robot.

Our aim is to evolve the role of a social humanoid robot in the caregiving workflow from a tool that is manually controlled during therapy sessions to an autonomously functioning technological support tool. In particular, when a BD manifestation is detected from sensors installed in the nursing home or from wearables worn by the residents, the robot should drive to the resident, and generate an audio-visual stimulus to temporarily distract the resident and possibly alert the staff if the BD manifestation is not stopped.

A key component of such a system - and the main contribution of this paper - is a control algorithm that pro- 
actively positions a social robot in a nursing home in order 119 to minimize the Time-To-Intervention (TTI) for a maximum number of BD manifestations. We define the TTI as the time elapsed between the detection of a BD from the sensor data and the robot having approached the resident close enough to start an intervention. We evaluate this algorithm with simulations based on realistic datasets annotated by the staff of closed dementia wards in two ${ }_{124}$ nursing homes in Belgium. Our use case imposes the following specific requirements:

1. Single robot Social robots are an expensive invest-127 ment for nursing homes which are, in Belgium as128 well in many other countries, under financial pressure. ${ }_{129}$ The controller algorithm was thus primarily designed ${ }_{130}$ for a single robot, leaving extensions to multi-robot ${ }_{131}$ deployments for future work.

2. Location priority Although some patterns can be observed in the daily displacements of a resident, the $\mathrm{e}_{134}$ occurrence of a $\mathrm{BD}$ is a stochastic process related $\mathrm{to}_{135}$ the individual and not to a spatial position such $\mathrm{as}_{136}$ a common room or the bed room. These patterns ${ }_{137}$ change over time, e.g. when a new resident arrives af- ${ }_{138}$ ter the decease of a resident (caregivers report typical ${ }_{130}$ stays of 9-15 months), or if the frequency of BDs of $\mathrm{a}_{140}$ resident increases as dementia progresses. Therefore, ${ }_{141}$ the robot should adapt its position to the actual loca- ${ }_{142}$ tion of the residents, weighted for the statistical $\mathrm{BD}_{143}$ risk profile of each resident.

3. Trajectory As BD events are detected using wear- ${ }_{145}$ ables or sensors, the main design goal for the robot's $\mathrm{s}_{146}$ trajectory in the nursing home is to arrive as fast $\mathrm{as}_{147}$ possible to a resident if such an event occurs - not to ${ }_{148}$ patrol and detect as much BDs as possible. If no res- ${ }_{149}$ ident moves significantly - a situation not uncommon ${ }_{150}$ in a nursing home - the optimal robot position does ${ }_{15}$ not change and the robot can stay idle.

4. Finite duration $\mathrm{BD}$ events have a finite duration, ${ }_{153}$ meaning that a $\mathrm{BD}$ can only be alleviated if a robot ${ }_{154}$ arrives within a fixed duration after the onset of a BD. ${ }_{155}$

5. Energy management Since the system is intended $\mathrm{as}_{156}$ a support tool in the caregiving workflow, the amount ${ }_{157}$ of actions required from the caregivers must be as minimal as possible. The robot should timely move ${ }^{158}$ itself to a charger location. Preferably, charging cycles ${ }^{159}$ are scheduled when fewer BD events are expected.

The rest of this paper is structured as follows. In sec-162 tion 2, we relate existing studies on robot positioning al-163 gorithms to the specific requirements imposed by BD in-164 terventions. In section 3 we situate this positioning algo- 165 rithm in a broader cyber-physical framework that contains 166 components for BD detection, positioning and navigation. 167 In section 4 , we explain our control algorithm that deter-168 mines the position and charging times of the robot. $\operatorname{In}_{169}$ section 5 , we evaluate the performance of our pro-active 170 position control algorithm. We conclude the paper in sec-171 tion 6 .

\section{Related work}

Continuous surveillance of a target environment by a robot has a wide range of applications such as property surveillance, environment monitoring in disaster sites and ecological monitoring (for an extensive review, see e.g. Nigam (2014)). Our studied problem of a robot positioning itself nearby locations of interest relates to robotic surveillance. In this section, we evaluate existing work on the requirements that were listed in section 1. A summary can be found in Table 1 .

In many of the works in this domain, the robot cycles through a predetermined sequence of locations. The aim is to balance dwell time, i.e. time spent at each location, with the time between two visits of the same location, see e.g. Smith et al. (2012), Yu et al. (2017)). For our use case, fixed patrolling circuits are less efficient since the trajectories followed by residents may vary from day to day. Yu et al. (2015) and Baykal et al. (2016) study a problem where events are generated according to a location-specific Poisson process. The trajectory and dwell times of a single robot are optimized to register as much events as possible. The events considered in these works are instantaneous: if the robot is not at the location when an event occurs, it is completely missed by the robot. The goal of our control algorithm is however to maximize the number of BDs alleviated (i.e. robot arrives no longer than a time $T$ after the onset of a $\mathrm{BD}$ ) and not to maximize the number of $\mathrm{BD}$ events detected, since $\mathrm{BD}$ detection is performed by sensors and wearables.

Battery management is often accounted for in terms of a constraint, ensuring that the robot arrives at a charging point (just) before running out of battery. For instance, Mersheeva and Friedrich (2015) calculate trajectories for a set of unmanned aerial vehicles where batteries can only be replaced at a limited set of locations. Rather than maximizing the length of a single trajectory between subsequent charging cycles, we let the robot charge its battery in idle times such that it can alleviate more BDs whenever they do occur.

In Tran et al. (2017), three different technologies are compared to solve the planning and scheduling problem of deploying multiple robots in a nursing home environment. Multiple robots need to jointly fulfill a set of tele-presence sessions, reminding residents about the upcoming Bingo activity as well as leading the Bingo activity. At the beginning of each day, a new task schedule is generated for each robot. Our position control algorithm is not designed for such deadline and location-constrained announcement and interaction tasks. Instead, we position the robot nearby residents so that the robot can quickly reach them if a resident starts exhibiting a BD. Finding an robot schedule for the combination of pre-scheduled and stochastically arriving interaction tasks is an interesting topic for future research. 


\begin{tabular}{|c|c|c|c|c|c|}
\hline & $\begin{array}{l}\text { Single or } \\
\text { multi-agent }\end{array}$ & Trajectory & $\begin{array}{l}\text { Energy } \\
\text { management }\end{array}$ & Location priority & Event type \\
\hline Smith et al. (2012) & Multi & $\begin{array}{l}\text { Predetermined static } \\
\text { cycle }\end{array}$ & None & $\begin{array}{l}\text { Dwell times } \\
\text { based on } \\
\text { accumulation } \\
\text { function }\end{array}$ & None \\
\hline $\begin{array}{l}\text { Mersheeva and } \\
\text { Friedrich (2015) }\end{array}$ & Multi & $\begin{array}{l}\text { Sequence over } \\
\text { landmarks, with } \\
\text { replacement of } \\
\text { batteries at base } \\
\text { stations }\end{array}$ & $\begin{array}{l}\text { Charge when } \\
\text { critically low }\end{array}$ & $\begin{array}{l}\text { Predetermined } \\
\text { stationary } \\
\text { location priorities }\end{array}$ & None \\
\hline Yu et al. (2015) & Single & $\begin{array}{l}\text { Periodically updated } \\
\text { sequence over } \\
\text { landmarks, without } \\
\text { revisits }\end{array}$ & None & $\begin{array}{l}\text { Rate of events } \\
\text { (stationary) }\end{array}$ & Instantaneous \\
\hline Baykal et al. (2016) & Single & $\begin{array}{l}\text { Periodically updated } \\
\text { sequence over } \\
\text { landmarks, with } \\
\text { possible revisits }\end{array}$ & None & $\begin{array}{l}\text { Rate of events } \\
\text { (non-stationary) }\end{array}$ & Instantaneous \\
\hline Tran et al. (2017) & Multi & $\begin{array}{l}\text { Planned based on } \\
\text { resident day schedule }\end{array}$ & $\begin{array}{l}\text { Charge times are } \\
\text { included in plan }\end{array}$ & $\begin{array}{l}\text { Fixed day } \\
\text { schedule for } \\
\text { resident }\end{array}$ & None \\
\hline Yu et al. (2017) & Single & $\begin{array}{l}\text { Fixed sequence over } \\
\text { landmarks, no } \\
\text { revisits }\end{array}$ & None & $\begin{array}{l}\text { Time since last } \\
\text { visit and dwell } \\
\text { time }\end{array}$ & None \\
\hline Ours & Single & $\begin{array}{l}\text { Dynamic positioning } \\
\text { on grid }\end{array}$ & $\begin{array}{l}\text { Charge in idle } \\
\text { times or when } \\
\text { necessary }\end{array}$ & $\begin{array}{l}\text { Risk-weighted } \\
\text { person tracking }\end{array}$ & Finite duration \\
\hline
\end{tabular}

Table 1: Comparison of the proposed framework with current state of the art on the requirements for BD intervention introduced in section 1.

\section{Background}

The positioning algorithm is part of a complete framework for personalized interventions of social robots, developed in the WONDER project ${ }^{1}$. In this section, we delineate the responsibilities of the positioning algorithm in the framework and briefly discuss the other building blocks that have been realized in the project.

Our system was designed in co-creation with the staff of closed dementia wards in two nursing homes. In such a ward, residents have their private bedroom while meals and leisure activities are organized in common rooms. For reasons of personal safety, residents cannot leave the ward unaccompanied.

The framework can be seen as a cyber-physical system that receives constant input from sensors and wearables on the residents and that steers a social robot throughout the premises of the nursing home: corridors, common rooms and private bedrooms. The conceptual architecture is illustrated in Figure 1.

The data processing block continuously tracks the residents' locations and whether they start exhibiting a behavioral disturbance (BD), e.g. by monitoring sound level to detect yelling or by tracking walking patterns to detect

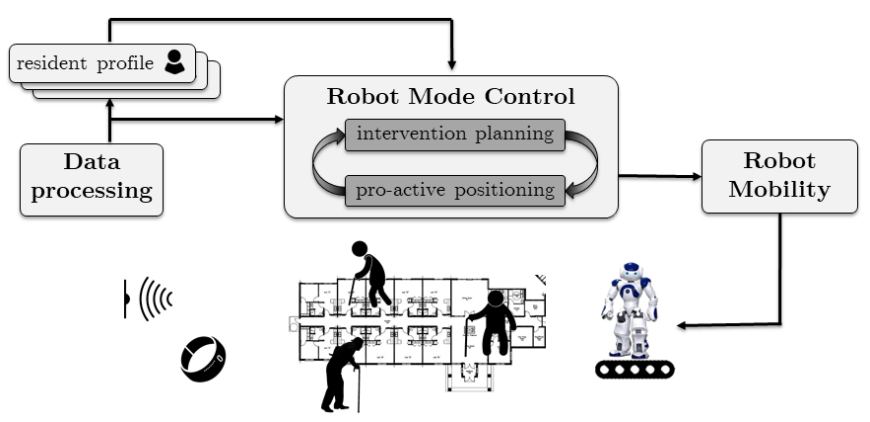

Figure 1: The social robot alternates between a pro-active mode and an intervention mode, based on real-time sensor data as well as historical information. The Robot Mobility block controls navigation through the corridors, common rooms and (private) bedrooms.

\footnotetext{
${ }^{1}$ https://www.imec-int.com/en/what-we-offer/ research-portfolio/wonder
} 
wandering behavior. Such BD detection algorithms have for instance been reported in (Lin et al., 2018; Vuong et al., 2015; Beltrán et al., 2014).

The robot control alternates between a pro-active mode and an intervention mode. In the default pro-active mode, the robot is positioned close to where the most BD are expected in the near future. As we will detail in the rest of this paper, these estimations are based on the combination of historical information on BD occurrences for each resident with real-time location information. For instance, during the night the robot will position itself closer to the bedrooms, while in the afternoon it will be close to a common room where all residents are gathered.

If a BD is detected, the system switches to the intervention mode. The robot drives towards a particular resident to attempt to alleviate the $\mathrm{BD}$ by starting an interaction. The success of the robot intervention can be monitored again via the wearables and sensors and if necessary, a caregiver is alerted for further assistance.

\subsection{Personalized interactions}

In co-creation with the nursing home staff (Ongenae ${ }_{253}$ et al., 2017), we defined a number of intervention actions ${ }_{254}$ that the robot can perform when in front of a resident: 255 asking a question about an important event in his lifetime, 256 reading out a news highlight and playing a song. Both the ${ }_{257}$ style and content of the intervention are tailored to the $\mathrm{e}_{258}$ resident. Style parameters include the language, speak-259 ing volume and approaching distance. The robot can play ${ }_{260}$ a favorite song, tell a short story about a topic of interest (e.g a news headline) or elicit a positive memory from ${ }_{261}$ the resident's lifetime. All these robot actions, together ${ }_{262}$ with profile information, are formally described in an ontology. Modeling this information in an ontology allows to ${ }_{264}$ incorporate knowledge from other domains, for instance, ${ }_{265}$ notions of an elderly's culture that determine the style ${ }_{266}$ of greeting (Bruno et al., 2017) or the way of approaching (Truong et al., 2016). By following and interpreting ${ }_{26}$ the links in the ontology, it can be automatically derived ${ }_{269}$ which profile information can be used to personalize the ${ }_{270}$ action. The effort of the care staff is limited to inputting ${ }_{271}$ some basic profile information about interests, language, ${ }_{272}$ etc. This is information that is already captured in today' $s_{273}$ care practices.

\subsection{Mobile platform for the Nao robot}

In our study, we used the humanoid Nao robot. The same robot was used in the KSERA project (Torta et al., ${ }^{275}$ 2014). The obtained results in a senior center home sug- ${ }^{276}$ gest that the participants did not feel any anxiety during ${ }^{277}$ interaction, mostly due to the shape and small size of the ${ }^{278}$ robot.

It is important for the robot to move fluently through- ${ }^{280}$ out the nursing homes. The Nao robot is however a legged ${ }^{281}$ robot that walks too slowly for our needs as it is limited to a speed of roughly $10 \mathrm{~cm} / \mathrm{s}$. To enable a more swift movement between locations in the nursing home, a new mobile
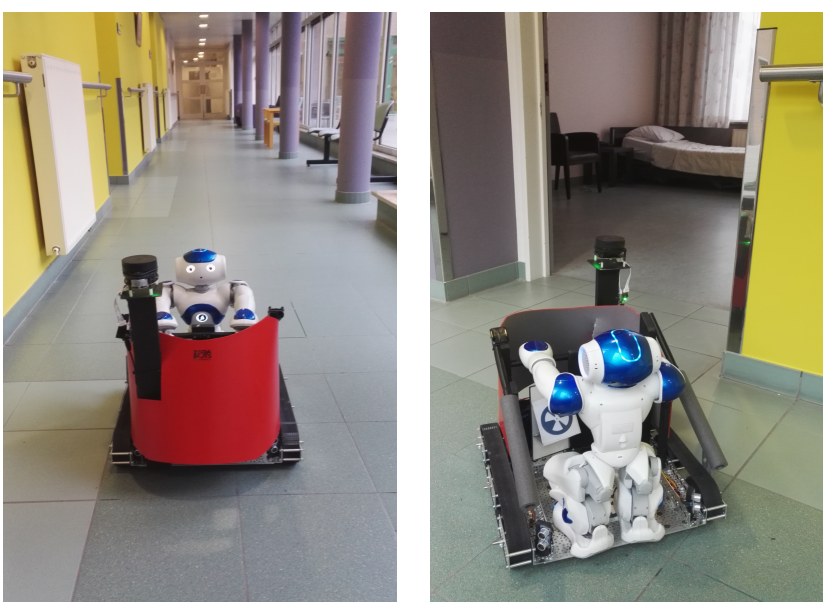

Figure 2: Front and backside view of the platform used by the social robot to move efficiently through the nursing home. The robot can autonomously step on and off the platform.

platform has been designed, shown in Figure $2^{2}$. The platform's mobility is accomplished by continuous track tires which are actuated by two independent motor drivers. We have equipped it with 5 simple sonars as well as a RoboPeak A1 lidar. The platform has a top speed of $1 \mathrm{~m} / \mathrm{s}$ making it much more suitable for a nursing home environment. The red color for the front was advised by the nursing home staff as red is the color that is most likely to be noticed by the persons with dementia.

\subsection{Study design}

Real sensor data was collected in two nursing homes located in Ghent, Belgium. Although we realized an integrated prototype of the framework in which a robot enters a room and initiates a personalized intervention (see Figure 2), we were not able to organize field trials to validate the positioning algorithm presented in this paper. The main reason is that the newly designed mobile platform was implemented as a proof-of-concept but was not certified for experimenting with humans in real environments. Hence, the experimental validation of the positioning algorithm is based on simulations with real data on BD manifestations in a dementia ward.

\section{Robot Position Control}

Residents constantly move between their room, the common areas and the corridors in the ward. If a BD is detected from sensor data, the robot will plan a personalized intervention. If no resident is currently exhibiting a BD, we aim to position the robot so as to minimize the time-tointeraction (TTI), which we define as the time between the detection of a BD and the robot arriving at the resident's

${ }^{2} \mathrm{~A}$ video of the robot driving around can be found at https: //vimeo.com/243125775. 


\begin{tabular}{|c|c|}
\hline \multicolumn{2}{|l|}{ Algorithm 1 Control routine } \\
\hline 1: while true do & 318 \\
\hline 2: $\quad$ if has_to_charge( () then & 319 \\
\hline navigate_to $\left(\vec{x}_{\text {charger }}\right)$ & 320 \\
\hline wait_until_charged() & 321 \\
\hline else if can_alleviate_bd () then & 322 \\
\hline navigate_to $\left(\vec{x}_{B D}\right)$ & 323 \\
\hline execute_personalized_interaction() & 324 \\
\hline else & 325 \\
\hline do_proactive_action() & 326 \\
\hline 10: $\quad$ end if & 327 \\
\hline 11: end while & 328 \\
\hline
\end{tabular}

current location and start a personalized intervention sce- ${ }_{33 .}$ nario. As we will show in section 5.1, our empirical data $a_{332}$ collected in two nursing homes reveals temporal patterns ${ }_{33}$ in the type of BD exhibited, e.g. there are typically fewer f $_{334}$ BDs during organized activities or meals. Therefore the ${ }_{335}$ control algorithm will constantly steer the robot towards $s_{336}$ locations in the ward where there is a high probability of $\mathrm{a}_{337}$ resident exhibiting a BD. Our algorithm is designed such $_{338}$ that it can be deployed in any nursing home (e.g. with dif $_{339}$ ferent day schedule) or with changing population charac- ${ }_{340}$ teristics (new residents, progressively declining residents). ${ }_{341}$ Lastly, the robot should never run out of out battery so the $3_{342}$ control algorithm must also decide when the robot should $_{343}$ move to a charging station deployed in the nursing home. ${ }_{344}$

\subsection{Environment model}

We define the nursing home as a two-dimensional $\operatorname{grid}_{346}$ $X$ wherein our robot as well as all residents are at discretized time $t$ positioned at one of the possible positions ${ }^{347}$ $\vec{x}=(x, y) \in X$ on the grid. Example grids of the two nurs-348 ing homes participating to our study are shown in Fig. 3.349 We denote the battery level of the robot at time $t$ as $b(t)^{350}$ $(0<b(t)<100)$. There is a single charger placed at 351 location $\vec{x}_{\text {charger }} \in X$.

At any moment in time, the robot is either standing 353 idle (on a non-charger position), charging, moving (with a constant speed $v$ ) or interacting with a resident. The corresponding depletion of the battery per time step, $\Delta b$, is modeled as fractions of a base discharge per timestep $c$ :

$$
\Delta b= \begin{cases}c \cdot f_{\text {idle }} & \text { if idle } \\ -c \cdot f_{\text {charge }} & \text { if charging } \\ c \cdot f_{\text {move }} & \text { if moving } \\ c \cdot f_{\text {int }} & \text { if interacting }\end{cases}
$$

The actions of the robot are controlled by a continuously running control algorithm, of which the pseudo-code is displayed in Alg. 1. Every iteration of this control algo-358 rithm starts with a check of the current battery level of the 359 robot. The function has_to_charge() (line 2 of Alg. 1) com-360 putes the distance $D\left(\vec{x}_{\text {robot }}, \vec{x}_{\text {charger }}\right)$ between the current 361

robot position and the location of the charger and checks if the current battery level is still sufficient for reaching the charger, i.e. if $b(t)>D\left(\vec{x}_{\text {robot }}, \vec{x}_{\text {charger }}\right) \cdot c \cdot f_{\text {move }}+B_{\text {min }}$, with $B_{\min }$ a safety margin.

As distance measure $D(x, y)$ we take the $A^{*}$-distance (Hart et al., 1968). If the battery level is not high enough then the robot will immediately go towards the charger. By performing this check we ensure that our robot never runs out of battery, which would require a manual intervention of the staff. Please note that once an robot decides it must charge, it will always fully charge. We have chosen to do this in order to avoid pivoting behavior and shall further motivate this choice after explaining the cost functions in more detail (see section 4.3).

If the robot does not need to charge, the control routine checks if the algorithms analyzing the sensor data report a BD ongoing at one of the residents (line 5 of Alg. 1) and if it can alleviate them depending on the location of the reported BD, the robot's current position and its battery level. It again checks this by computing the power necessary to $(i)$ travel towards the location of the ongoing $\mathrm{BD},(i i)$ alleviate the ongoing $\mathrm{BD}$ and (iii) travel from the $\mathrm{BD}$ location to the charger. If the robot cannot alleviate the $\mathrm{BD}$ due to energy constraints, the ongoing $\mathrm{BD}$ will be ignored.

If there are no ongoing BDs and the robot has sufficient battery, we execute a cost-based optimal control problem (line 9 of Alg. 1) to optimize the robot position (in terms of TTI). The internals are detailed in the next section.

\subsection{Pro-active action control}

In pro-active mode, the robot can take five possible actions $a \in \mathcal{A}$ : moving one grid cell up/down/left/right or stay on the current location (idle). The optimal anticipative action for the robot depends on the relative position of the robot towards the charger and towards all residents, the probability of all residents to exhibit a BD in the near future, and the actual battery level $b(t)$.

We define our state space as:

$$
\begin{aligned}
S=\left\{s(t)=\left(\vec{x}_{\text {robot }}, \vec{x}_{\text {resident }}, b(t), t\right) \mid \vec{x}_{\text {robot }} \in X,\right. \\
\left.\vec{x}_{\text {resident }} \in X, b \in[0,100]\right\}
\end{aligned}
$$

We consider movement of our robot to be noiseless and 

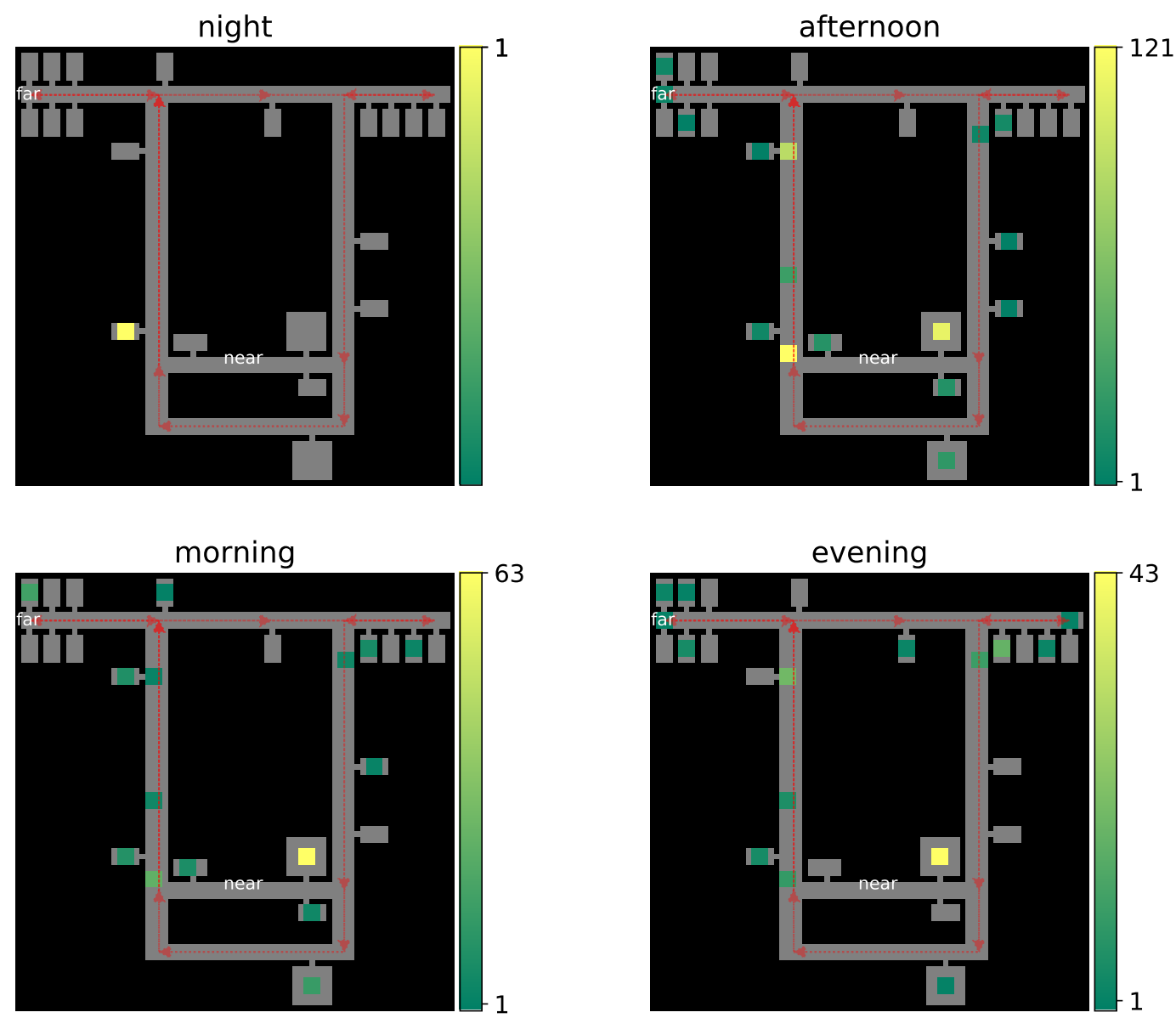

(a) Nursing Home 1
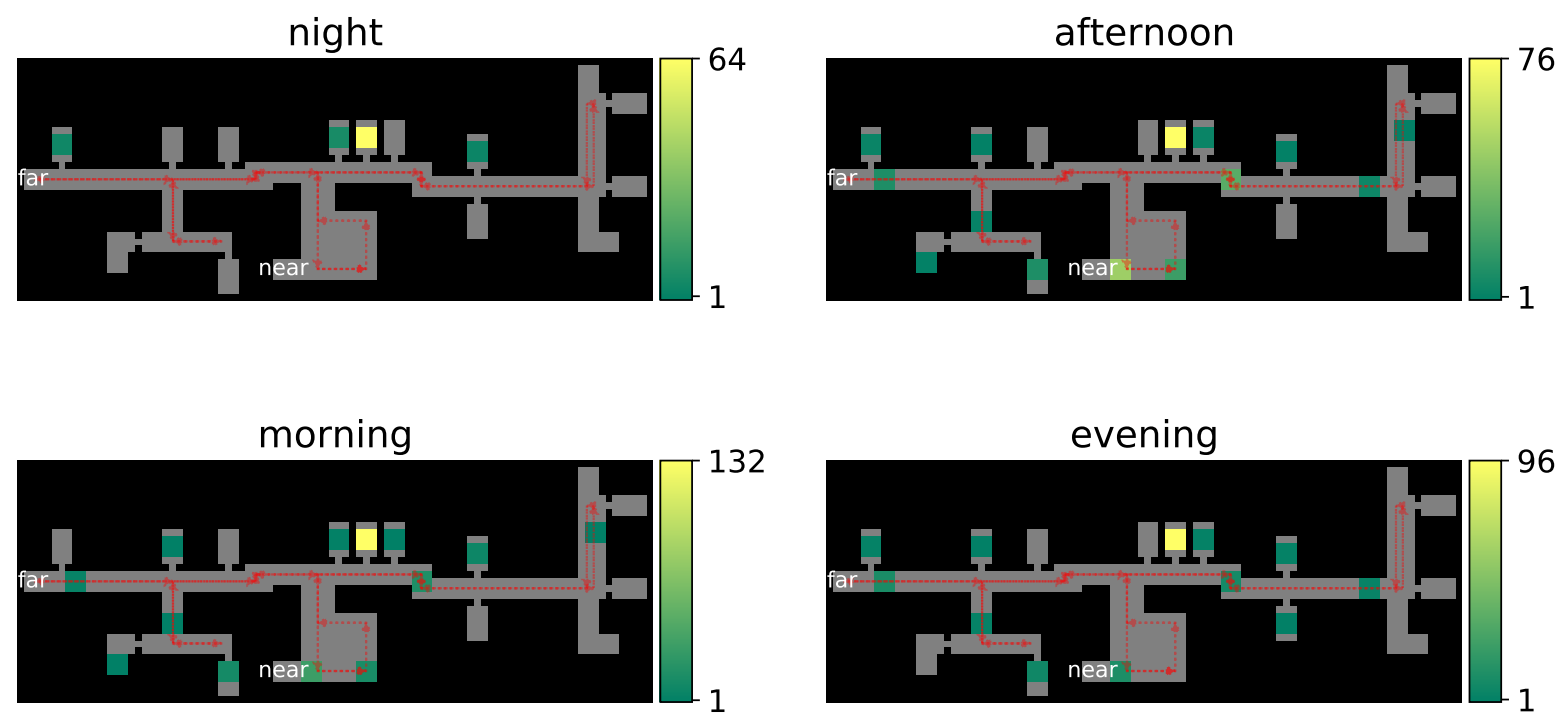

(b) Nursing Home 2

Figure 3: Heatmap of the locations where BDs are taking place in the nursing homes. Accessible areas are coloured gray while inaccessible ones are black. Small rooms on the outer rim of the grids are patient rooms while large rooms indicate common rooms. The plans are reduced versions of the original floor plans because rooms of patients for which no informed consent of the patient was obtained are also marked as inaccessible. Colours indicate the number of behavioral disturbances collected over 100 days for nursing home 1 and 135 days for nursing home 2. The red dotted line is the patrolling path for the patrolling baseline. The night is defined between 22:00 and 06:00, the morning between 06:00 and 12:00, the afternoon between 12:00 and 18:00 and the evening between 18:00 and 22:00. 
The cost linked with the environment, $C_{\text {env }}$ is intro-398 duced to ensure that our robot does not take an impossibles99 action by for example walking into a wall.

$$
C_{\text {env }}(\vec{x})= \begin{cases}\infty & \text { if } \vec{x} \text { is infeasible/occupied } \\ 0 & \text { otherwise }\end{cases}
$$

\subsubsection{Battery cost}

Each action draws a certain amount of energy from the battery, which is accounted for by assigning a cost to each action. Furthermore the battery cost $C_{b a t}$ contains a linear cost for the distance to the charger. This part ensures that if the other terms in the total cost function are zero then our robot is steered towards the charger. The cost term $C_{b a t}$ thus becomes:

$C_{\text {bat }}(s, a)=C_{\text {charger }}\left(\vec{x}_{\text {robot }}\right)+C_{\text {action }}(a)$

where $C_{\text {charger }}\left(\vec{x}_{\text {robot }}\right)=D\left(\vec{x}_{\text {robot }}, \vec{x}_{\text {charger }}\right)$

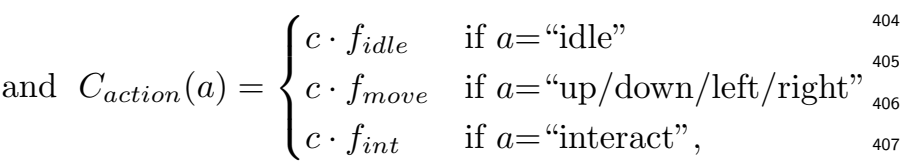

This cost term competes with the cost $C_{B D}$ reflecting ${ }^{409}$ the anticipated BDs. The charger will thus become an ${ }^{410}$ attractive position for the robot if very few BDs are anti-411 cipated in the near future.

\subsubsection{BD anticipation cost}

At any idle moment, and if the battery level is sufficient, the robot should position itself close to the location where ${ }_{415}$ many residents are gathered that have a high probability ${ }_{416}$ of exhibiting a $\mathrm{BD}$ in the near future. We therefore assume ${ }_{417}$ the availability of a predictor function $\mu\left(r, t, t_{\mu}\right)$ which $\mathrm{re}^{-417}$ turns the probability that resident $r$ will exhibit one or more BDs in the interval $\left[t, t+t_{\mu}\right]$, with $t$ the current time ${ }_{420}$ and $t_{\mu}$ a lookahead-time that indicates how far our robot ${ }_{421}$ should look into the future for anticipating BDs. This pre- ${ }_{422}$ dictor function can be based on advanced machine learn- ${ }_{423}$ ing, however, as we will show in section 5, we also obtain ${ }_{424}$ good results with a simple daily updated historical profile. ${ }_{425}$ The cost term for BD anticipation is then given by weigh- ${ }_{426}$ ing the distance between the robot and all residents with $_{427}{ }_{426}$ the predicted BD likelihood for that resident:

$$
C_{B D}(s, a)=\sum_{\mathrm{r}} \mu\left(\mathrm{r}, t, t_{\mu}\right) D^{2}\left(\vec{x}_{\text {robot }}, \vec{x}_{\text {resident }}\right)
$$

In Eq. (5), $D$ is again the $A^{*}$-distance measure on a grid The distance is squared such that decreasing the distance 434 from the robot to a location with future predicted BDs435 decreases this cost term more than how $C_{b a t}$ is reduced by 436 moving the robot towards the charger. This ensures that ${ }_{437}$ if there are residents with predicted BDs between $t$ and $t+t_{\mu}$ the robot is steered towards them in favor of going to the charger.

We scale the lookahead-time $t_{\mu}$ with the battery level $b$ such that the time window decreases in size as the battery level decreases. Resulting from this comes the intuitive result that steering towards the charger (due to the linear battery cost) becomes more attractive as the battery decreases since the number of expected BDs (and thus the cost term $C_{B D}$ ) also decreases due to a smaller time window. Specifically, the time window is set equal to

$$
t_{\mu}(s)=\max \left(0, t_{\text {depl }}(b)-\hat{t}(s)\right)
$$

where

$$
\hat{t}(s)=t_{\vec{x} \rightarrow \vec{x}_{\text {charger }}}(s)+t_{\text {fullcharge }}(b)+t_{\text {alleviateBD }}(s)
$$

where $t_{d e p l}(b)$ denotes the time needed for the battery to deplete fully, $t_{\vec{x}_{\text {robot }} \rightarrow \vec{x}_{\text {charger }}}$ the time needed to travel to the charger from the current position, $t_{\text {fullcharge }}$ the time needed to fully charge from the current battery level and $t_{\text {alleviateBD }}$ the time needed to $(i)$ travel to the nearest resident, ( $i i)$ alleviate a BD at that location and (iii) travel from that location to the charger. Intuitively, Eq. (6) reflects that we should not look for BDs expected in the far future if we have only a limited battery level. In the case where the battery level decreases below a certain point we have $\hat{t} \geq t_{d e p l}$ and thus $t_{\mu}=0$ at which the cost $C_{B D}$ vanishes. The battery cost $C_{b a t}$ is then minimized by steering the robot towards the charger as desired.

\subsection{Avoiding pivoting behavior}

The different terms of the cost function try to attract the robot either to the charger or to other positions in the grid. As introduced in section 4.1, the robot always charges to full in case it decides to charge. One might wonder why a robot could not leave the charger to alleviate a BD without being charged to full. Early experimentations showed however that without imposing the constraint to charge to full, the robot might start constantly pivoting between two positions on the grid. Consider for example the case that our robot is at a low battery level. Since its battery level is low, the lookahead-time is small and the predictor functions $\mu($.$) will indicate a low probability. However, as its$ battery charges the lookahead time increases accordingly and BDs might be anticipated. Since the battery level has increased by positioning itself on the charger, the robot is steered again towards the expected BD. As soon as the robot leaves the charger position its battery level drops, thus decreasing its lookahead-time again and the anticipated BD might disappear outside of its time window $t_{\mu}$. In this case, the robot again decides to steer towards the charger and the process repeats once its battery level rises again slightly. The result is that our agent will constantly pivot between charging and going towards an optimal location while its battery will reach a certain level. The robot 
will only take steps on and off the charger position and 489 will be unable to alleviate any BD. In order to prevent 490 this undesired behavior we have chosen to let the robot 491 always charge to full if it positions itself on the charger 492 while its battery is not fully charged.

\section{Evaluation}

In this section, we evaluate the control routine that was ${ }^{497}$ presented in Alg. 1. We will compare with two baselines: ${ }^{498}$

- a reactive controller, called return, that will always500 steer the robot to the charger if there is no BD ongo-501 ing. If there is a BD ongoing (and the robot is not502 in an intervention), the controller will always move ${ }^{503}$ the robot to the resident, unless there is insufficient504 battery. Essentially, this comes down to nullifying the505 term $C_{B D}$ while keeping the rest of Alg. 1 unmodi-500 fied.

- a patrolling controller, called patrolling, will make the robot follow a fixed and predefined path through the ${ }_{510}$ hallways of the nursing home. Whenever a BD occurs, ${ }_{511}$ the robot will move to that resident. After alleviating ${ }_{512}$ the $\mathrm{BD}$, the robot resumes the predetermined trajec- ${ }_{513}$ tory. The robot moves only to the charger when the ${ }_{514}$ battery becomes critically low. The predefined paths $5_{515}$ in the two nursing homes under study are displayed $_{516}$ in Fig. 3.

The main figures of merit for our pro-active position ${ }^{51}$ controller and the two baselines are i) the number of $\mathrm{BD}^{519}$ interventions that the robot can carry out by optimizing its ${ }^{520}$ charging cycles, and ii) the Time-To-Interception (TTI) ${ }_{5_{22}}^{{ }^{21}}$ The number of BDs alleviated reflects the global perfor- ${ }^{522}$ mance of our controller, i.e. it shows the potential number ${ }^{523}$ of BDs that could be alleviated by the robot. The $\mathrm{TTI}^{524}$ reflects the performance of our framework as perceived by ${ }_{526}^{525}$ an individual resident, i.e. how long does it take before the ${ }^{526}$ robot arrives.

\subsection{BD dataset collection and analysis}

To get better insight in the frequency and type of 530 BD manifestations, the staff of two nursing homes in 531 Ghent, Belgium registered during a period of approx. threes32 months via a smartphone app every time they observed533 yelling or wandering behavior for any of the participants534 for which informed consent was obtained. The data col-535 lection procedure, the observational studies and the pro-536 totype deployment of the mobile platform have been ap-537 proved by the ethical committee of UZ Brussels. PatientS5з8 (or their legal representative) and nursing home staff have5s9 given their informed consent prior to the start of the study.540 In nursing home 1, 15 residents were monitored, of which ${ }_{541}$ 1 deceased and 2 dropped out during the registration pe-542 riod. This resulted in 877 registrations of a wandering el-543 derly and 106 registrations of an elderly yelling. In nursing ${ }_{544}$ home 2, 10 residents were monitored, and the staff registered 564 times yelling behavior and 279 times wandering behavior.

These staff annotations were linked to an estimation of the resident's actual location. To track the out-of-room movement, gateways with Bluetooth Low Energy (BLE) support were installed in several hallways and common living rooms. During the day, most residents are in the common living rooms (CLR), whereas in the evening people tend to spend more time in their room or in the hallway. Residents participating to the study received a Xiami Mi Band ${ }^{\circledR}$ wearable or an $X{ }^{\circledR}$ BLE tag was attached to their wheelchair or walker. With these wearables we were able to track the location of the participants.

Pro-active positioning of the robot is only meaningful if $\mathrm{BD}$ events are not randomly spread over time and space. In Fig. 4, we show how the annotated BD observations are distributed over the day. As intuitively expected, a clear drop is seen during the night. We also have less annotations during meal times. One should however be very careful to conclude that there are less BDs during meal times, since this observation may also be caused by the staff being too busy to annotate the BDs. Even if the drop in BDs can be attributed to this effect, one could argue that robot interventions during meal times are anyway not desired since the staff is nearby the residents.

In order to check the underlying spatial patterns of the collected data we have plotted a heatmap of the locations where the annotated BDs took place, for every part of the day, over a time period of 100 days for nursing home 1 and 135 days for nursing home 2. For nursing home 1 we see that there are almost no BDs during the night while in the morning the location of BDs is relatively widely spread. In the afternoon most BDs are located in the common rooms while in the evening most BDs take place in or close by the rooms of the patients. Note however that in nursing home 1 most of the BDs are located in the large room around the bottom right. In nursing home 2 we see that a single room is accounting for most of the BDs.

\subsection{Experiment configuration}

We evaluate our position control algorithm and the two baseline controllers in a simulator that shifts time while replaying the mobility and BD occurrences per patient. At each time stamp, all cost terms are calculated. To determine the BD anticipation cost $C_{B D}$ (Eq. (5)), we need the BD predictor functions $\mu($.$) . These were constructed by$ binning daytime in 15 minutes intervals and incrementing the counter of the appropriate bin if a BD was annotated. Generation of this array is additive throughout the simulation: i.e. after simulating one day, the $\mu($.$) were updated$ with the corresponding annotations of that day. This allows us to gradually build up knowledge on the patterns, and to account for new residents in the nursing home. In the current experiments, we applied no windowing, i.e. all historical data available until a particular day is taken into account. 


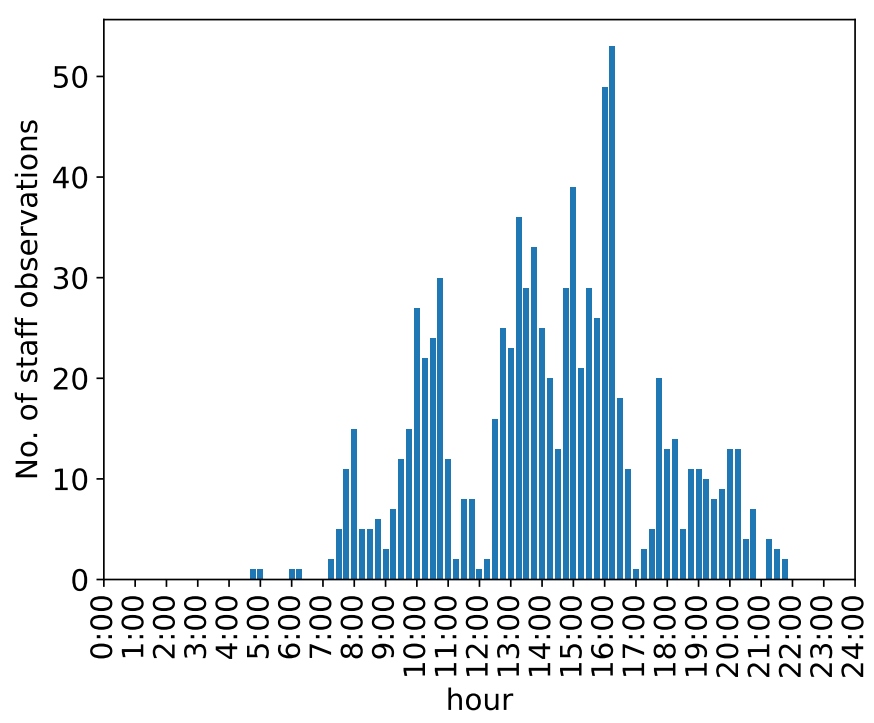

(a) Nursing Home 1

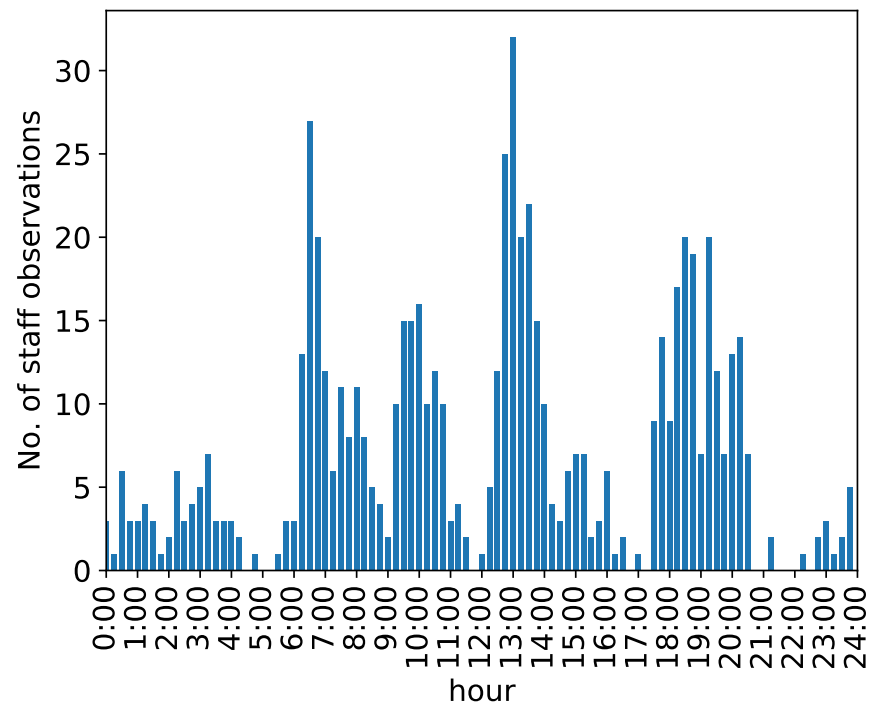

(b) Nursing Home 2

Figure 4: Number of observed BDs for the two different nursing homes for patients who gave ethical consent. Both show the observations in time slots with a length of 15 minutes.

We wish to verify optimal parameters for our framework578 in which our robot alleviates as many BDs as possible while 579 keeping the TTI as low as possible. The values of the base 580 discharge rate $c, f_{\text {idle }}$ and $f_{\text {charge }}$ (see Eq. (1)) were kept581 constant in all experiments, reflecting a discharge time of $f_{52}$ 3 hours for a full battery in idle time, and a maximum ${ }_{583}$ charge time of 1 hour. We set the time of one BD allevia-584 tion interaction to 3 minutes, which we deem is a represen-585 tative duration for the type of personalized interventions586 we designed (Ongenae et al., 2017), such as asking a short ${ }_{587}$ question or playing a song.

The main experimental parameter is the robot velocity ${ }^{589}$ $v$. Obviously, if the velocity is high the robot can reach ${ }^{590}$ a resident exhibiting a BD very fast from any location. ${ }^{591}$ Hence, we expect that the differences between our pro- ${ }^{592}$ active control framework and both return and patrolling ${ }^{593}$ baselines will become smaller with increasing $v$. However note in practice the maximum speed is limited by safety ${ }^{594}$ considerations and/or mechanical constraints.

The position of the charger, $\vec{x}_{\text {charger }}$ is our second exper-506 imental parameter. As shown in Fig. 3 and as explained in597 section 5.1, in both nursing homes we could identify a se-598 lect number of locations where most of the BDs are taking ${ }_{599}$ place. Obviously, if the charger is located far from from600 these regions, the robot must spent more energy on driving 601 to and from the charger. We experimented with two (ex-602 treme) positions of the charger for each nursing home, one 603 in a common room where most of the BDs were annotated, 604 and one at a far end of the nursing home. These positions, 605 labelled 'near' and 'far', are indicated in Fig. 3. Note that606 in practice, safety concerns restrict the possible charger ${ }_{607}$ locations, e.g. it can not be placed in front of a fire exit.608 Also, the spatial BD distribution might shift over time due 609 to changes in the population or the progression of dementia. If the charger is located far away from these regions where most BDs take place the return heuristic is at a bigger disadvantage because the robot will be constantly on the move. When movement is costly this makes the battery drain even faster and much time is wasted on driving to and from the charger. The patrolling baseline is also at a disadvantage whenever the BDs occur far away from the current position on the patrolling path, wasting much time on patrolling in areas which are of less importance. Our cost-based control framework however should position the robot (idle) at optimal positions and only charge when deemed beneficial (i.e. in idle times) or when necessary. On the other hand, if the charger is located near the region where most BDs take place the difference in performance between the frameworks should decrease.

\subsection{Number of BDs alleviated}

We first wish to verify that our framework alleviates as many BDs as possible. For this we plot $\alpha$, which equals the total number of alleviated BDs divided by the total number of BDs, versus the velocity $v$.

As seen in Fig. 5, the number of BDs that the robot can alleviate increases with the velocity since the robot loses less time with navigating to and from the charger position. This observation is further backed up by comparing with the return controller: for low velocities the number of alleviated BDs is low if the charger is positioned far away from where the majority of the BDs take place (right panels in the subfigures). In these situations the robot is spending a lot of time to move to and from the charger position. Our cost-based framework improves the number of alleviated BDs since the robot is only moved towards the charger 


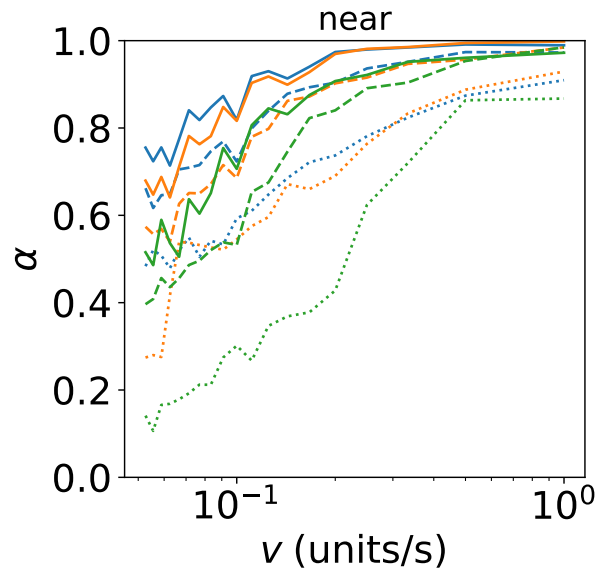

(a) Nursing Home 1
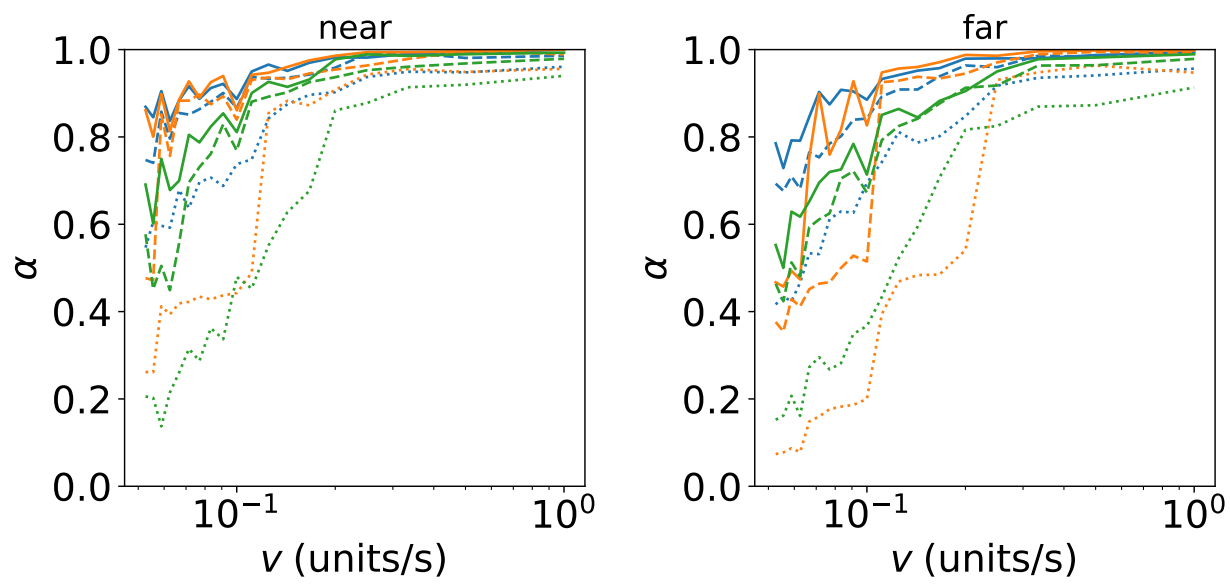

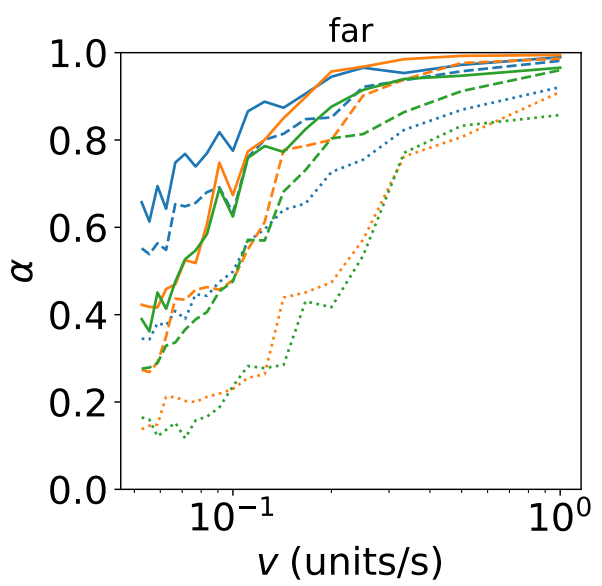

pro-active, $t_{B D}=300$

pro-active, $t_{B D}=600$

pro-active, $t_{B D}=1800$

return, $t_{B D}=300$

return, $t_{B D}=600$

return, $t_{B D}=1800$

patrolling, $t_{B D}=300$

patrolling, $t_{B D}=600$

patrolling, $t_{B D}=1800$

(b) Nursing Home 2

Figure 5: The number of alleviated BDs divided by the total number of BDs as a function of the robot's velocity. The labels 'near' and 'far' on top of each graph indicate the charger position $\vec{x}_{\text {charger }}$. Results are plotted for $\left(f_{\text {idle }}: f_{\text {move }}: f_{\text {int }}\right)=(1: 3: 2)$, BD duration $=10$ min.

in idle times (or unless it is absolutely necessary). The 630 improvement in alleviated BDs for lower velocities is seen in both nursing homes. Note that the results in Figure $5_{631}$ denote a configuration where the battery cost of movement, $f_{\text {move }}$, is larger than the cost of interacting, $f_{\text {int }} .{ }^{632}$ We believe this is the most common setting. However, for ${ }^{633}$ completeness, we have included in Appendix A the results ${ }^{634}$ when $f_{\text {int }}$ is larger than $f_{\text {moving }}$.

The dataset only contains the time of annotation, but $_{637}^{636}$ not the duration of the BD manifestations or the interaction time necessary to alleviate a BD. We have therefor ${ }_{639}$ run simulations with different durations of BDs and evalu- ${ }^{639}$ ated the sensitivity of the number of BDs alleviated. The results can be seen in Figure 5. The results confirm the ${ }_{641}^{641}$ intuitive expectation that if a $\mathrm{BD}$ has a longer duration, ${ }_{643}$ the number of BDs alleviated will increase since the robot ${ }^{643}$ has a longer time window to intervene.

The BD duration sets an upper limit to the TTI. In the 646 experiments reported in the next subsection we will set ${ }_{647}$ the BD duration to 10 minutes since it represents average 648 performance of the robot.

\subsection{Time-To-Interception}

Next we measure the TTI as a function of the robot velocity, under the same conditions of charger positions and battery cost ratio's as in the previous section. The results are plotted in Fig. 6. (see Appendix A for additional figures for different cost fractions; the conclusions are similar).

In all conditions, the TTI decreases with increasing velocity since the robot can move faster from any location towards the location of a BD manifestation. By comparing the results for charger positions $\mathrm{A}$ and $\mathrm{B}$, it can be seen that in both nursing homes the charger position has a very limited effect on the TTI achieved by all controllers. This means that, when considering the TTI, the staff can freely choose where to place the charger station so that it does not block fire exits or frequently visited hallways.

In most experiment conditions, the patrolling baseline has the worst TTI of all three controllers. This was ex- 

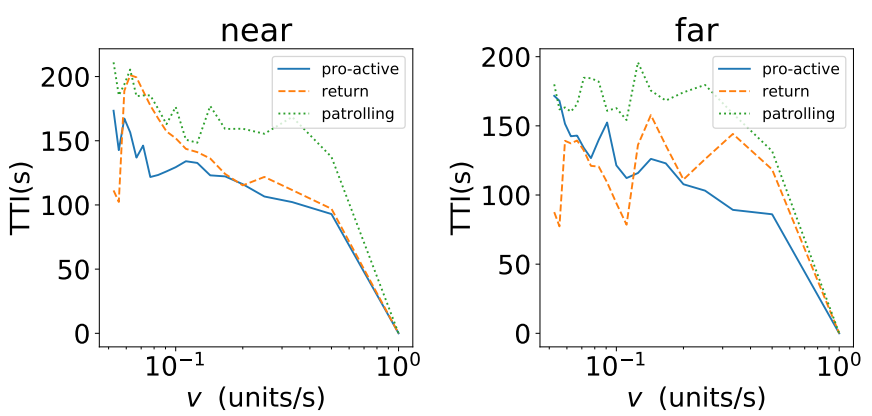

(a) Nursing Home 1
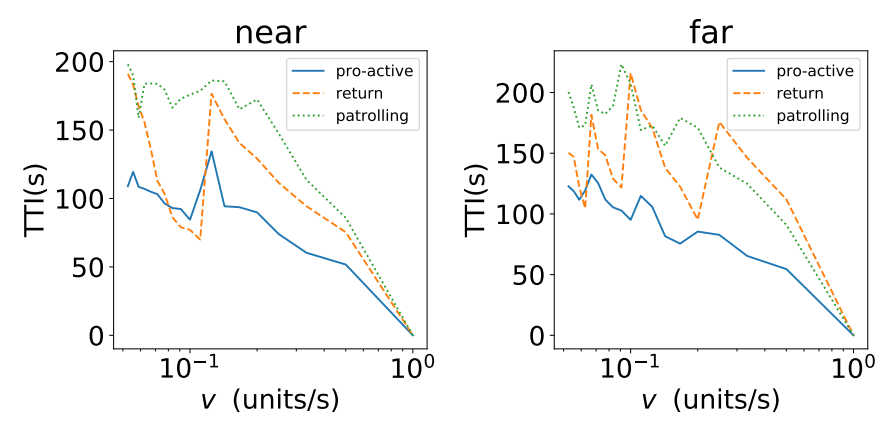

(b) Nursing Home 2

Figure 6: TTI in seconds as a function of robot velocity, for different cost ratio's and different charger positions. Results are plotted for $\left(f_{\text {idle }}: f_{\text {move }}: f_{\text {int }}\right)=(1: 3: 2)$, BD duration of 10 minutes.

pected, since the robot is following a trajectory throughout 688 the entire nursing home and does not account for the ac-689 tual resident location. Our pro-active positioning results ${ }_{690}$ in the lowest TTI in most experiment conditions. Note ${ }_{691}$ that the TTI is sometimes reduced by more than a minute ${ }_{692}$ for lower velocities, which is a significant result considering 693 that the BDs have a duration of 10 minutes. The influence ${ }_{694}$ of the ratio of battery costs of different actions is minimal. ${ }_{695}$

Only for lower velocities and charger position B, the 696 return controller achieves lower TTIs. This better perfor- ${ }_{697}$ mance might seem counterintuitive, since charger position ${ }_{698}$ B was chosen as far as possible from locations with fre- ${ }_{699}$ quent $\mathrm{BD}$ events. However, these results must be inter- ${ }_{700}$ preted in combination with Figure 5(a), where it can be ${ }_{701}$ seen that the return controller achieves the lowest num- ${ }_{702}$ ber of interventions. The return controller thus intervenes ${ }_{703}$ very fast in a very small number of BD events.

Since our predictor functions $\mu($.$) accumulate knowledge { }^{704}$ over time, we have also evaluated if the average TTI de- ${ }_{706}$ creases when more data becomes available. In Figure $7_{707}^{700}$ we plot the average TTI for each simulated day (see Appendix A for additional figures for different cost fractions). We have calculated the p-value of the coefficient of a linear regression function through the data against the null hypothesis of a zero coefficient (meaning that the number of ${ }_{712}^{711}$ days has no effect on the obtained TTI). Although we obtain negative slopes for our pro-active position controller in all experiment conditions, only for nursing home $2 \mathrm{we}_{715}$ obtain p-values $<0.05$ to reject the null hypothesis.

\section{Conclusion and Future Work}

In this paper, we have outlined the different algorithms ${ }^{720}$ and control strategies to pro-actively position a mobility-721 enhanced humanoid robot in order to rapidly approach a ar2 $^{2}$ person with dementia exhibiting a behavioral disturbance.723

The positioning controller presented in this paper can ${ }_{724}$ be refined in several ways. First, we do not account for 725 periods where the robot is used in leisure activities orga-726 nized by the caregivers (bingo, group singing, etc.). If the 727 planning of these activities is incorporated in the position 728 control algorithm, we can ensure that the robot is fully charged at the start of the activity.

Second, our algorithm is currently not accounting for the actual position of the caregiver staff. When a caregiver is nearby when a BD is detected, the robot should not be sent and instead move itself to areas where fewer personnel is available. Besides location tracking of the caregiver staff, adding such functionality would require advance activity recognition to determine if a caregiver is actually available to alleviate a BD.

Third, the predictor functions $\mu($.$) can be refined. Cur-$ rently the predictor functions are based on accumulating all occurred BDs. If the framework would be used on longer time scales, one need to remove the BDs from deceased residents, or one would have to investigate the progression rate of dementia, and if this translated in changes in BD manifestations. If sensor and wearable data can be analyzed in near real-time, one could even imagine a real-time BD risk assessment algorithm that continuously adjusts the predictor functions.

Fourth, the current framework is purely reactive: a robot interaction is only started in response to a BD event. We could extend the framework to also plan robot interactions outside of $\mathrm{BD}$ episodes. By having the robot interact more with residents, the robot could potentially contribute to an improvement of the Quality-of-Life and thus prevent BD occurrences. Such additional interactions would also mitigate the risk of exposing sensitive medical information, since in the current framework the proximity of the robot to users is an implicit signal that one or more persons are more likely to have a BD. However, planning such interventions outside of BD episodes will require sufficiently diverse and more elaborate interaction capabilities of the robot.

Lastly, longer term experiments are needed to evaluate the acceptance and effectiveness of the designed solution. Although the cognitive capabilities of residents in a closed room ward are severely degraded, we conjecture that the robot behavior must be sufficiently diverse to maintain an enjoying experience on the long term and have a significant impact on the Quality of Life. 

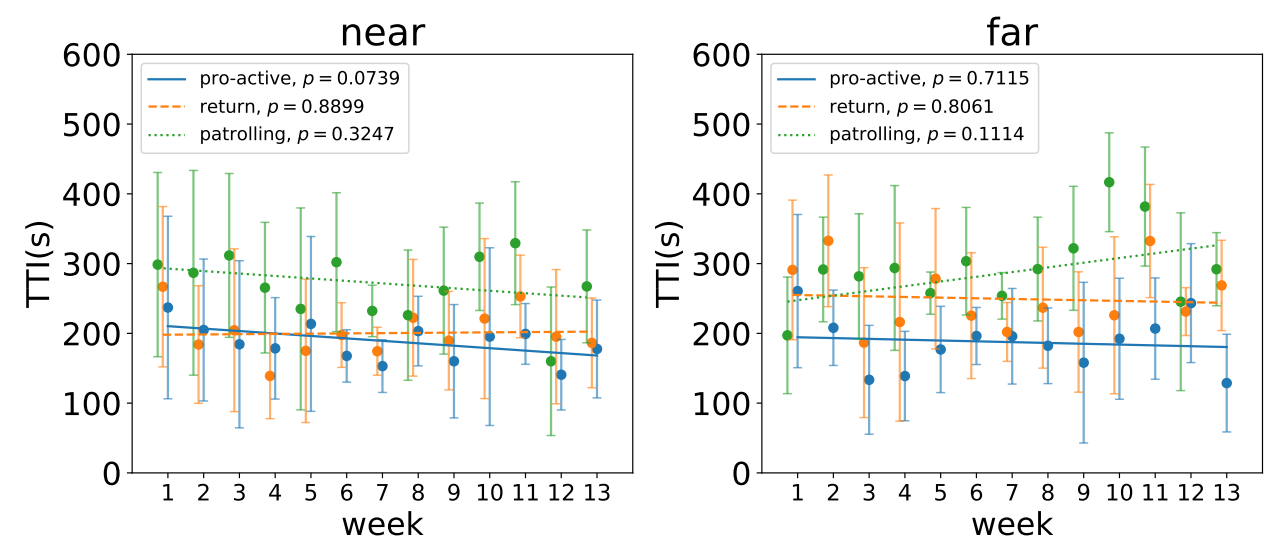

(a) Nursing Home 1
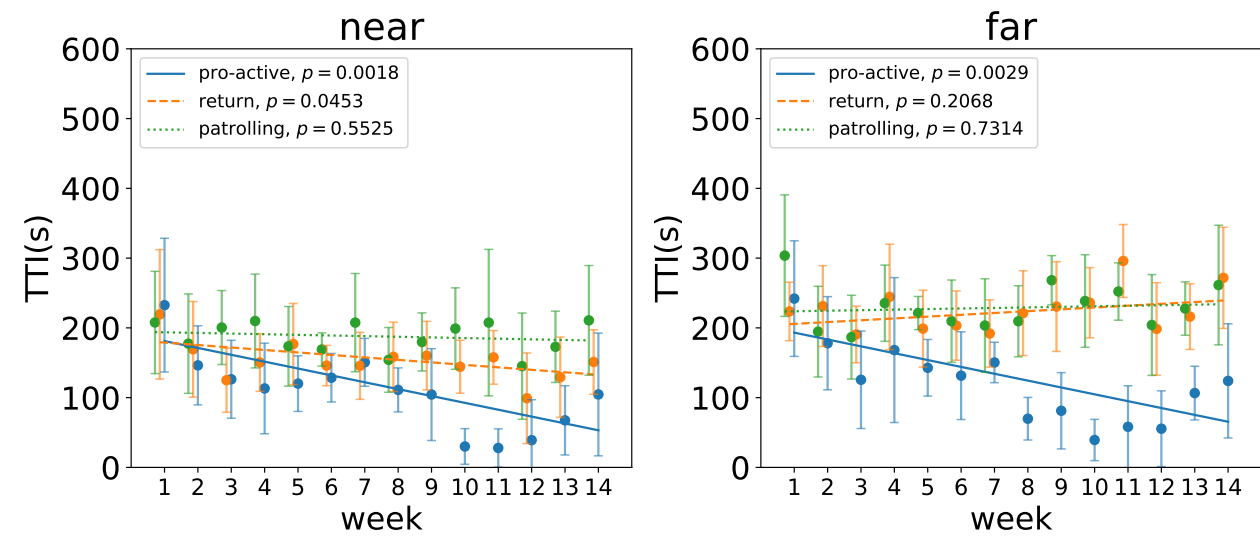

(b) Nursing Home 2

Figure 7: Average TTI of alleviated BDs per week, for charger positions 'near' and 'far'. Errorbars indicate standard deviation of the TTI. Results are plotted for $\left(f_{\text {idle }}: f_{\text {move }}: f_{\text {int }}\right)=(1: 3: 2), v^{-1}=6$, BD duration $=10 \mathrm{~min}$.

\section{Acknowledgements}

The imec.ICON WONDER project is realized in $\mathrm{col}_{-758}^{757}$ laboration with imec, with project support from $\mathrm{VLAIO}_{759}$ (Flanders Innovation and Entrepreneurship). The re-760 searchers would like to explicitly thank the residents $\operatorname{and}_{762}^{761}$ staff of the two participating nursing homes for their efforts ${ }_{763}^{762}$ and collaboration.

World Health Organization, Fact Sheet on Dementia, 2017 (accessed $_{765}^{765}$ June 18, 2018)

S. Harper, Economic and social implications of aging societies, Sci- $^{767}$ ence 346 (2014) 587-591.

E. Demir, E. Kseolu, R. Sokullu, B. eker, Smart home assistant ${ }_{770}^{769}$ for ambient assisted living of elderly people with dementia, Pro- ${ }_{771}$ cedia Computer Science 113 (2017) 609 - 614. The 8th International Conference on Emerging Ubiquitous Systems and Pervasive ${ }_{773}$ Networks (EUSPN 2017) / The 7th International Conference on ${ }_{774}$ Current and Future Trends of Information and Communication ${ }_{775}$ Technologies in Healthcare (ICTH-2017) / Affiliated Workshops. 776 C. Mller, S. Lautenschlger, G. Meyer, A. Stephan, Interventions to support people with dementia and their caregivers during the ${ }_{778}$ transition from home care to nursing home care: A systematic ${ }_{779}$ review, International Journal of Nursing Studies 71 (2017) $139-_{780}$ 152.

J. L. OSullivan, P. Gellert, B. Hesse, L.-M. Jordan, S. Mller, J.- ${ }_{782}^{781}$ N. Voigt-Antons, J. Nordheim, Exploring attitudes of healthcare ${ }_{783}$ professionals towards ict-based interventions for nursing home res- idents with dementia: a mixed-methods approach, Contemporary Nurse 0 (2018) 1-13. PMID: 29279003.

A. K. Desai, L. Schwartz, G. T. Grossberg, Behavioral disturbance in dementia, Current Psychiatry Reports 14 (2012) 298-309.

B. S. Husebo, C. Ballard, R. Sandvik, O. B. Nilsen, D. Aarsland, Efficacy of treating pain to reduce behavioural disturbances in residents of nursing homes with dementia: cluster randomised clinical trial, BMJ 343 (2011).

T. Hazelhof, L. Schoonhoven, B. van Gaal, R. Koopmans, D. Gerritsen, Nursing staff stress from challenging behaviour of residents with dementia: a concept analysis, International Nursing Review 63 (2016) 507-516.

C. H. Sadowsky, J. E. Galvin, Guidelines for the management of cognitive and behavioral problems in dementia, The Journal of the American Board of Family Medicine 25 (2012) 350-366.

A. M. de Oliveira, M. Radanovic, P. C. H. de Mello, P. C. Buchain, A. D. B. Vizzotto, D. L. Celestino, F. Stella, C. V. Piersol, O. V. Forlenza, Nonpharmacological interventions to reduce behavioral and psychological symptoms of dementia: a systematic review, BioMed research international 2015 (2015).

S. Sabanovic, C. C. Bennett, W. L. Chang, L. Huber, Paro robot affects diverse interaction modalities in group sensory therapy for older adults with dementia, in: 2013 IEEE 13th International Conference on Rehabilitation Robotics (ICORR), pp. 1-6.

N. Jøranson, I. Pedersen, A. M. M. Rokstad, C. Ihlebæk, Effects on symptoms of agitation and depression in persons with dementia participating in robot-assisted activity: a cluster-randomized controlled trial, Journal of the American Medical Directors Association 16 (2015) 867-873. 
K. Wada, T. Shibata, T. Musha, S. Kimura, Effects of robot therapy for demented patients evaluated by eeg, in: 2005 IEEE/RSJ International Conference on Intelligent Robots and Systems, pp. 1552-1557.

M. Valent Soler, L. Agera-Ortiz, J. Olazarn Rodrguez, C. Mendoza Rebolledo, A. Prez Muoz, I. Rodrguez Prez, E. Osa Ruiz, A. Barrios Snchez, V. Herrero Cano, L. Carrasco Chilln, S. Felipe Ruiz, J. Lpez Alvarez, B. Len Salas, J. Caas Plaza, F. Martn Rico, P. Martnez Martn, Social robots in advanced dementia, Frontiers in Aging Neuroscience 7 (2015) 133.

K. Inoue, N. Sakuma, M. Okada, C. Sasaki, M. Nakamura, K. Wada, Effective application of palro: A humanoid type robot for people with dementia, in: K. Miesenberger, D. Fels, D. Archambault, P. Peñáz, W. Zagler (Eds.), Computers Helping People with Special Needs, Springer International Publishing, Cham, 2014, pp. 451-454.

N. Nigam, The multiple unmanned air vehicle persistent surveillance problem: A review, Machines 2 (2014) 13-72.

S. L. Smith, M. Schwager, D. Rus, Persistent robotic tasks: Monitoring and sweeping in changing environments, IEEE Transactions on Robotics 28 (2012) 410-426.

V. Mersheeva, G. Friedrich, Multi-uav monitoring with priorities and limited energy resources.

J. Yu, S. Karaman, D. Rus, Persistent monitoring of events with stochastic arrivals at multiple stations, IEEE Transactions on Robotics 31 (2015) 521-535.

C. Baykal, G. Rosman, K. Kotowick, M. Donahue, D. Rus, Persistent surveillance of events with unknown rate statistics, in: Workshop on the Algorithmic Foundations of Robotics (WAFR).

T. T. Tran, T. Vaquero, G. Nejat, J. C. Beck, Robots in retirement homes: Applying off-the-shelf planning and scheduling to a team of assistive robots, Journal of Artificial Intelligence Research 58 (2017) 523-590.

X. Yu, S. B. Andersson, N. Zhou, C. G. Cassandras, Optimal dwell times for persistent monitoring of a finite set of targets, in: American Control Conference (ACC), 2017, IEEE, pp. 5544-5549.

Q. Lin, W. Zhao, W. Wang, Detecting dementia-related wandering locomotion of elders by leveraging active infrared sensors, Journal of Computer and Communications 6 (2018) 94.

N. Vuong, S. Chan, C. T. Lau, S. Chan, P. L. K. Yap, A. Chen, Preliminary results of using inertial sensors to detect dementia-related wandering patterns, in: Engineering in Medicine and Biology Society (EMBC), 2015 37th Annual International Conference of the IEEE, IEEE, pp. 3703-3706.

J. Beltrán, R. Navarro, E. Chávez, J. Favela, V. Soto-Mendoza, C. Ibarra, Detecting disruptive vocalizations for ambient assisted interventions for dementia, in: L. Pecchia, L. L. Chen, C. Nugent, J. Bravo (Eds.), Ambient Assisted Living and Daily Activities, Springer International Publishing, Cham, 2014, pp. 356-363.

F. Ongenae, F. De Backere, C. Mahieu, S. De Pestel, J. Nelis, P. Simoens, F. De Turck, Personalized robotic intervention strategy by using semantics for people with dementia in nursing homes, in: ESWC2017, the Extended Semantic Web Conference; Lecture Notes in Computer Science, pp. 21-30.

B. Bruno, N. Y. Chong, H. Kamide, S. Kanoria, J. Lee, Y. Lim, A. Kumar Pandey, C. Papadopoulos, I. Papadopoulos, F. Pecora, et al., Paving the way for culturally competent robots: a position paper, in: 26th IEEE International Symposium on Robot and Human Interactive Communication (RO-MAN).

X. T. Truong, Y. S. Ou, T. D. Ngo, Towards culturally aware robot navigation, in: 2016 IEEE International Conference on Real-time Computing and Robotics (RCAR), pp. 63-69.

E. Torta, F. Werner, D. O. Johnson, J. F. Juola, R. H. Cuijpers, M. Bazzani, J. Oberzaucher, J. Lemberger, H. Lewy, J. Bregman, Evaluation of a small socially-assistive humanoid robot in intelligent homes for the care of the elderly, Journal of Intelligent \& Robotic Systems 76 (2014) 57-71.

P. E. Hart, N. J. Nilsson, B. Raphael, A formal basis for the heuristic determination of minimum cost paths, IEEE transactions on Systems Science and Cybernetics 4 (1968) 100-107. 

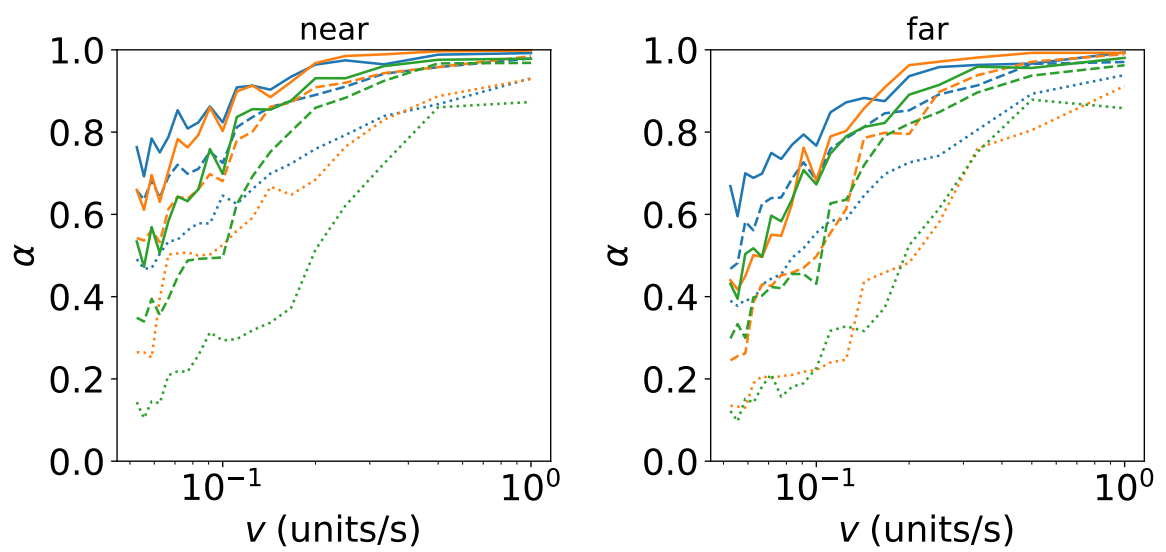

(a) $\mathrm{NH} 1$
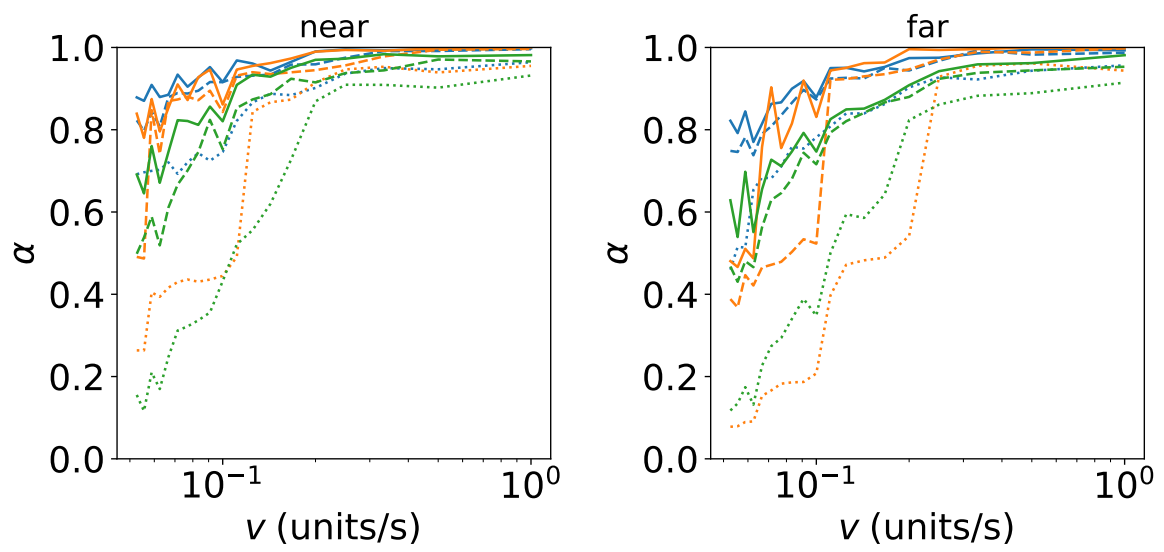

pro-active, $t_{B D}=300$

pro-active, $t_{B D}=600$

pro-active, $t_{B D}=1800$

return, $t_{B D}=300$

return, $t_{B D}=600$

return, $t_{B D}=1800$

patrolling, $t_{B D}=300$

patrolling, $t_{B D}=600$

patrolling, $t_{B D}=1800$

(b) $\mathrm{NH} 2$

Figure A.8: The number of attainable BDs divided by the total number of BDs versus the robots' velocity in units per second for both NH. The labels 'A' and 'B' on top of each graph refer to the charger position $\vec{x}_{\text {charger }}$. Results are plotted for $\left(f_{\text {idle }}: f_{\text {move }}: f_{\text {int }}\right)=(1: 2: 3)$

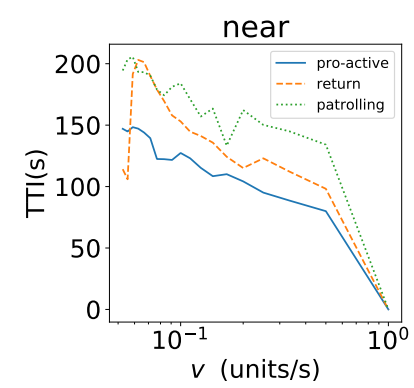

(a) NH1

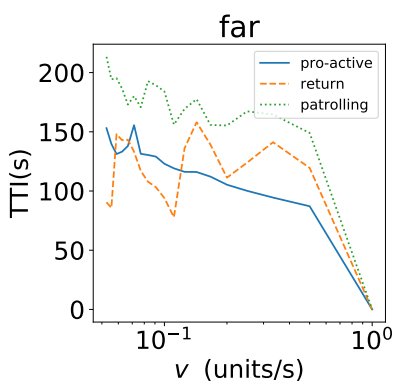

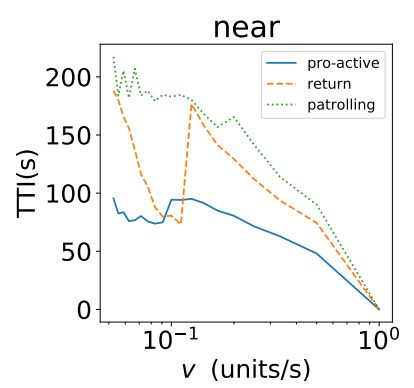

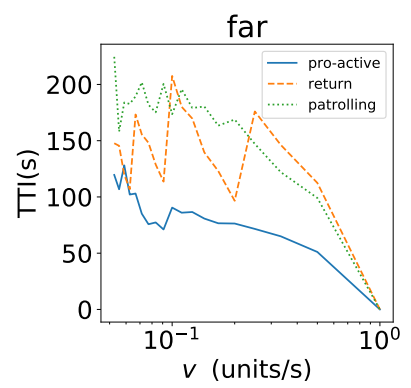

(b) $\mathrm{NH} 2$

Figure A.9: TTI in seconds as a function of robot velocity, for different cost ratio's and different charger positions. Results are plotted for $\left(f_{\text {idle }}: f_{\text {move }}: f_{\text {int }}\right)=(1: 2: 3), \mathrm{BD}$ duration $=10 \mathrm{~min}$. 

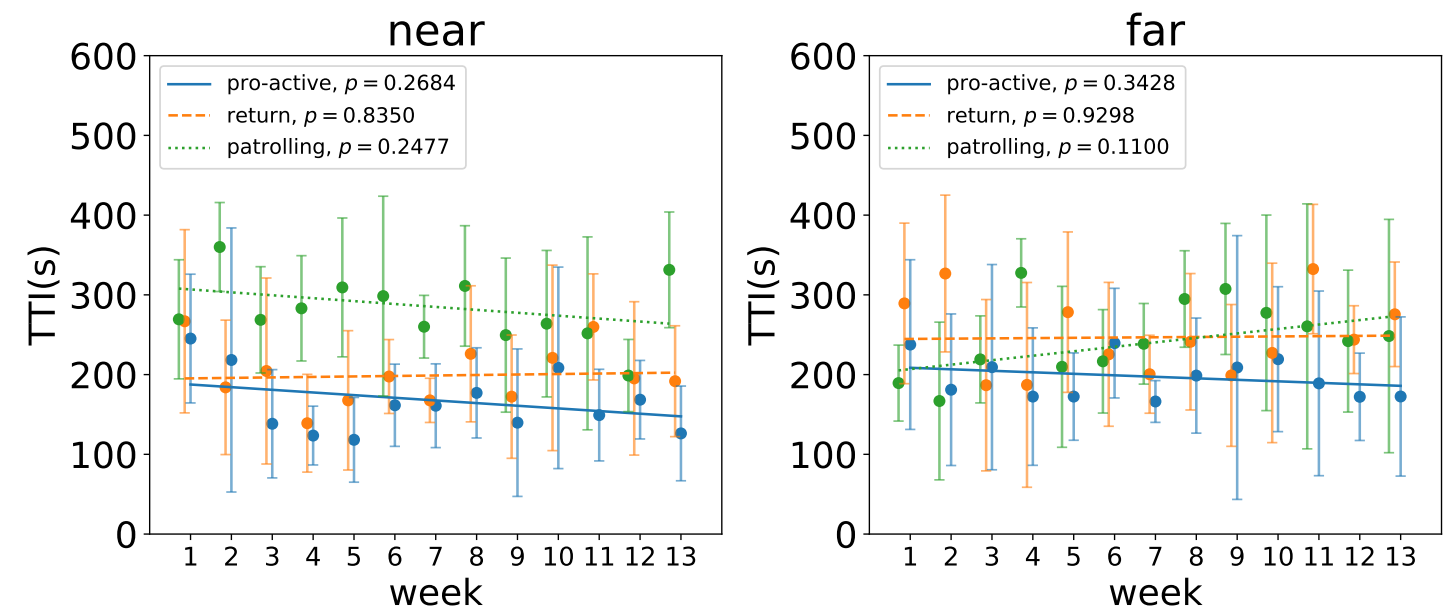

(a) NH1
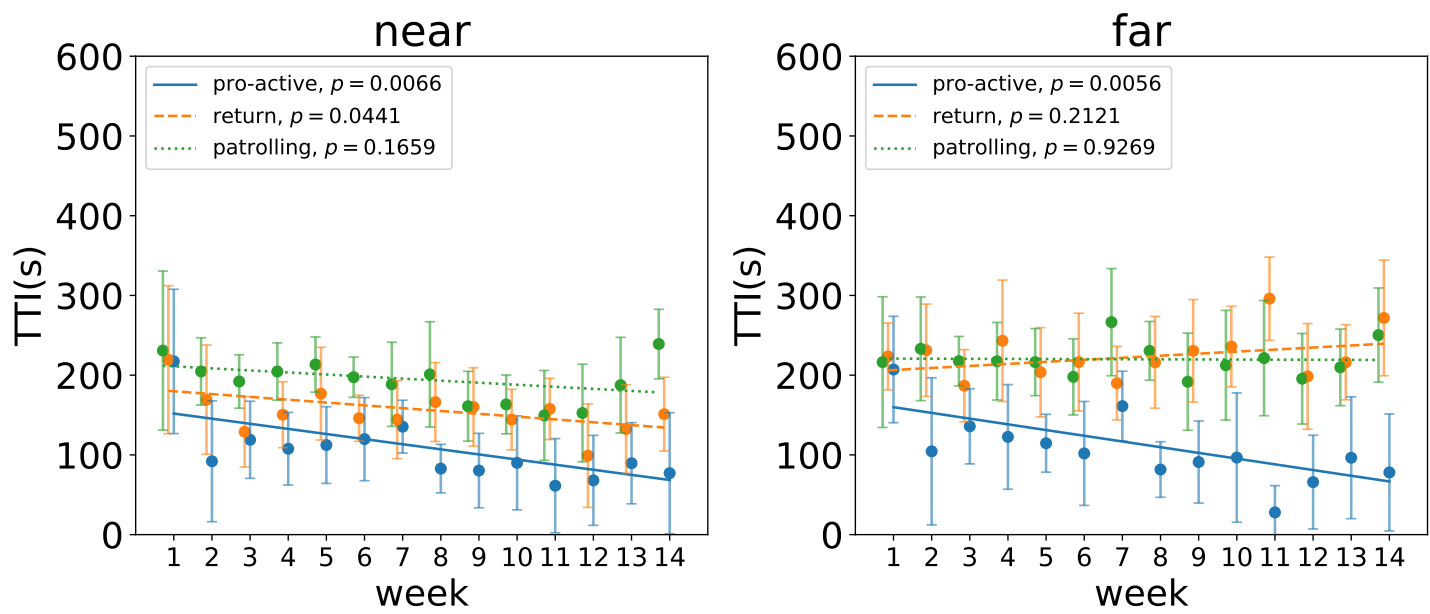

(b) NH2

Figure A.10: Linear fit of the TTI in seconds as a function of days simulated, for charger positions 'A' and 'B'. Errorbars indicate standard deviation of the TTIs of all alleviated BDs in one week. Results are plotted for $\left(f_{\text {idle }}: f_{\text {move }}: f_{\text {int }}\right)=(1: 2: 3), v^{-1}=6$, BD duration $=$ $10 \mathrm{~min}$. 